\title{
Rare disease research requires (and benefits from) global collaboration: three examples from the Castleman Disease Collaborative Network
}

\author{
Hayley Williamson ${ }^{1}$, Grant Mitchell ${ }^{1}$, Emily Zhen ${ }^{1}$, Auryn Enciso ${ }^{1}$, Samantha Kass Newman ${ }^{1,2}$, \\ Deise Ahagon', Michael Croglio ${ }^{1,3}$, Jason Ruth ${ }^{1,4}$, Raj Jayanthan ${ }^{1,5}$, Amy Yutong Liu', \\ Alexander Suarez ${ }^{1,6}$, David Fajgenbaum, ${ }^{1,6}$
}

Williamson H, Mitchell G, Zhen E, Enciso A, Newman SK, Ahagon D, Croglio M, Jayanthan R, Liu AY, Suarez A, Fajgenbaum. Rare disease research requires (and benefits from) global collaboration: three examples from the Castleman Disease Collaborative Network. Rev Med (São Paulo). 2016 July-Aug.;95(Special 3):24-9.

\begin{abstract}
Castleman disease (CD) describes a heterogeneous group of rare and poorly understood lymphoproliferative disorders that can involve flu-like symptoms, multiple organ system dysfunction, and even death. Prior to 2012, the limited and disparate resources, including clinical data, tissue samples, and research funding, prevented the medical community from making positive strides towards understanding the pathogenesis of CD. The Castleman Disease Collaborative Network (CDCN) was created in 2012 to accelerate research and drug development for CD by facilitating global collaboration, strategically investing in high impact research, and engaging patients throughout the research process. The CDCN's global collaborative network has been crucial for accelerating the understanding of CD. Together, the CDCN has already helped establish a uniform terminology system and a new model of pathogenesis. With the CDCN's network in place and an ambitious international research agenda, further breakthroughs for CD are on the horizon. In this paper, we report three examples of how global collaboration has helped to advance CD research, which we believe can serve as models for other research networks and international investigators.
\end{abstract}

KEYWORDS: Giant lymph node hyperplasia; Giant lymph node hyperplasia/pathology; Cooperative behavior; Castleman disease.

\section{BACKGROUND}

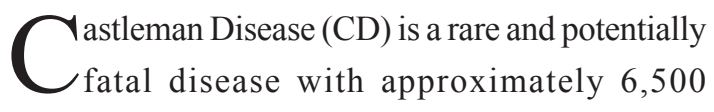

to 7,700 new cases diagnosed each year in the United States $^{1}$. CD describes a heterogeneous group of three lymphoproliferative disorders that range from a single region of enlarged lymph nodes with few symptoms (unicentric $\mathrm{CD}$ ) to multiple enlarged lymph nodes with flu-like symptoms, fluid accumulation, and even deadly multiple organ system failure (multicentric CD). Unicentric CD is often cured with surgical intervention ${ }^{2}$. Multicentric CD (MCD) is caused by excessive release of proinflammatory cytokines including interleukin-6 (IL-6) ${ }^{3}$. MCD is further subclassified into Human Herpes Virus-8 (HHV-8) associated MCD (HHV-8-associated MCD) and

1. Castleman Disease Collaborative Network, Philadelphia, PA, USA.

2. Department of Medicine, NYU Langone Medical Center, New York, NY, USA.

3. School of Medicine, Stony Brook University, Stony Brook, NY, USA.

4. Department of Medical Oncology, Dana-Farber Cancer Institute, Harvard Medical School, Boston, MA, USA.

5. School of Medicine, Meharry Medical College, Nashville, TN, USA.

6. Department of Medicine, Division of Translational Medicine \& Human Genetics, University of Pennsylvania, Philadelphia, PA, USA. Corresponding author: David C. Fajgenbaum, MD, MSc, MBA. Email: davidfa@mail.med.upenn.edu. 
HHV-8 negative or idiopathic MCD (iMCD) ${ }^{1}$. In HHV8 -associated MCD, the inflammatory cascade is caused by HHV-8 replication in B-cells, which release IL-6 and other pro-inflammatory cytokines ${ }^{4}$. Treatment of HHV-8associated MCD patients with rituximab, which depletes B-cells, is highly effective in these patients ${ }^{5}$. In iMCD, a similar inflammatory cascade occurs, but there is no HHV-8 and the cause of the inflammatory cascade, key immune cells, and key immune pathways remain unknown ${ }^{6}$. With an unknown etiology and pathogenesis, iMCD is difficult to treat, leading to an array of attempted treatments with variable success. A major breakthrough occurred in 2014 when siltuximab, a monoclonal antibody that neutralizes IL-6, became the first FDA-approved treatment for iMCD ${ }^{7}$. However, siltuximab only elicited a partial or complete response in 34 percent of patients in the clinical trial. Research is urgently needed to elucidate the pathogenesis of iMCD in order to identify treatments for the two-thirds of patients who do not respond to siltuximab ${ }^{7}$.

Like many rare diseases, there are several systemic factors that have slowed down progress for $\mathrm{CD}$ research and treatment. One major barrier for iMCD research was the lack of centralized clinical and research data. With no database to track treatments, physicians were left to make care decisions based on their own intuition, with little data to inform their choices ${ }^{8}$. Additionally, tissue samples were very difficult to locate for research as there was no centralized biorepository. The limited number of tissue samples for research has prevented investigators without access to samples from studying CD. ${ }^{8}$ The scarcity of tissue samples also contributed to the lack of federal funding for research. Since researchers did not have enough samples to generate sufficient preliminary data, they have not been in a position to apply for competitive federal funding. In fact, to date, iMCD researchers have never received NIH grants, which are a crucial funding source that other rare diseases have successfully leveraged ${ }^{8}$. Finally, researchers in different parts of the world were not using common terminology systems, so it was difficult to understand how research studies could build upon one another. It was clear that building a collaborative network was urgently needed to centralize clinical data and tissue samples, promote the sharing of samples to achieve larger sample sizes and generate preliminary data for federal funding, establish a unified terminology system, and drive forward an international research agenda.

In 2012, Dr. David Fajgenbaum joined together a team of fellow physicians, researchers, and patients to create the Castleman Disease Collaborative Network $(\mathrm{CDCN})^{8}$. The first phase of the $\mathrm{CDCN}$ was to create a collective global community that would work together in order to improve treatments and eventually cure $\mathrm{CD}^{8}$. Physicians and researchers from around the world were identified through PubMed searches and connected through an online discussion board and in-person meetings. An international Scientific Advisory Board (SAB) was established to prioritize research projects and set the overall direction of the organization'. The CDCN also supported and engaged patients and loved ones in the advancement of CD research ${ }^{9}$. See Figure 1 for a map demonstrating the distribution of physicians and researchers in the CDCN network from around the world today. See Figure 2 for a map demonstrating the distribution of patients in the CDCN network from around the word today.
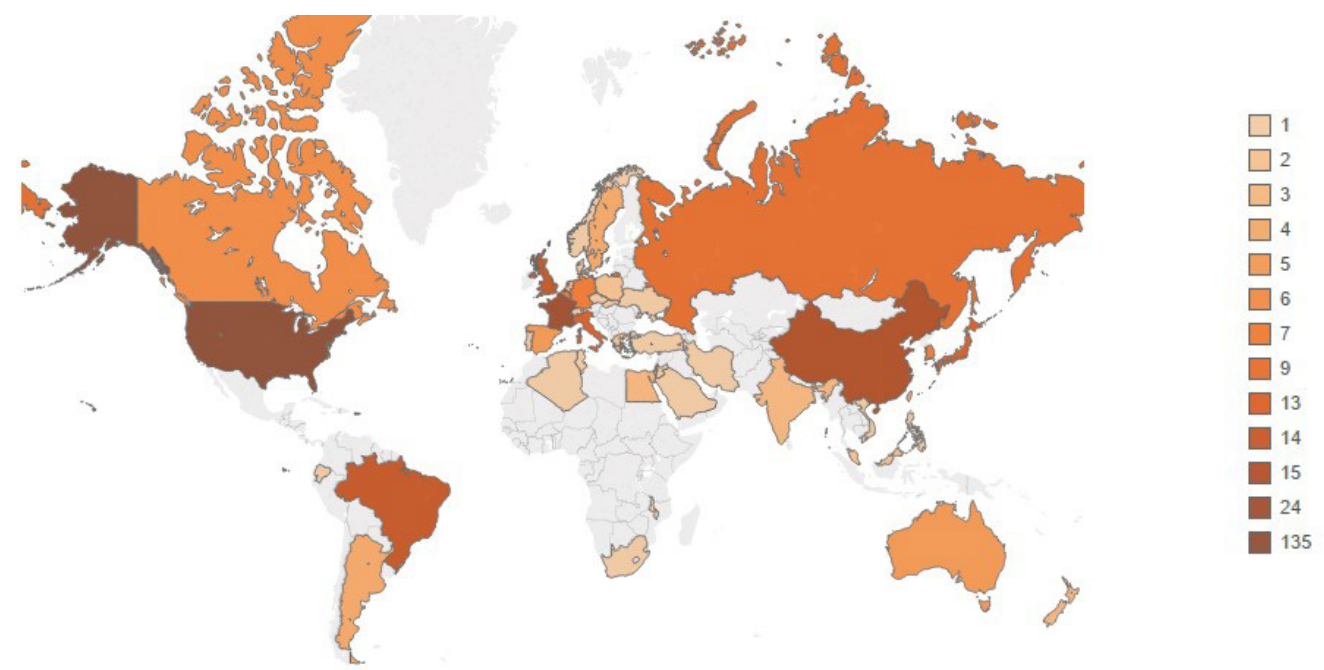

Figure 1. Worldwide distribution of the $317 \mathrm{CD}$ physicians and researchers connected through the CDCN 


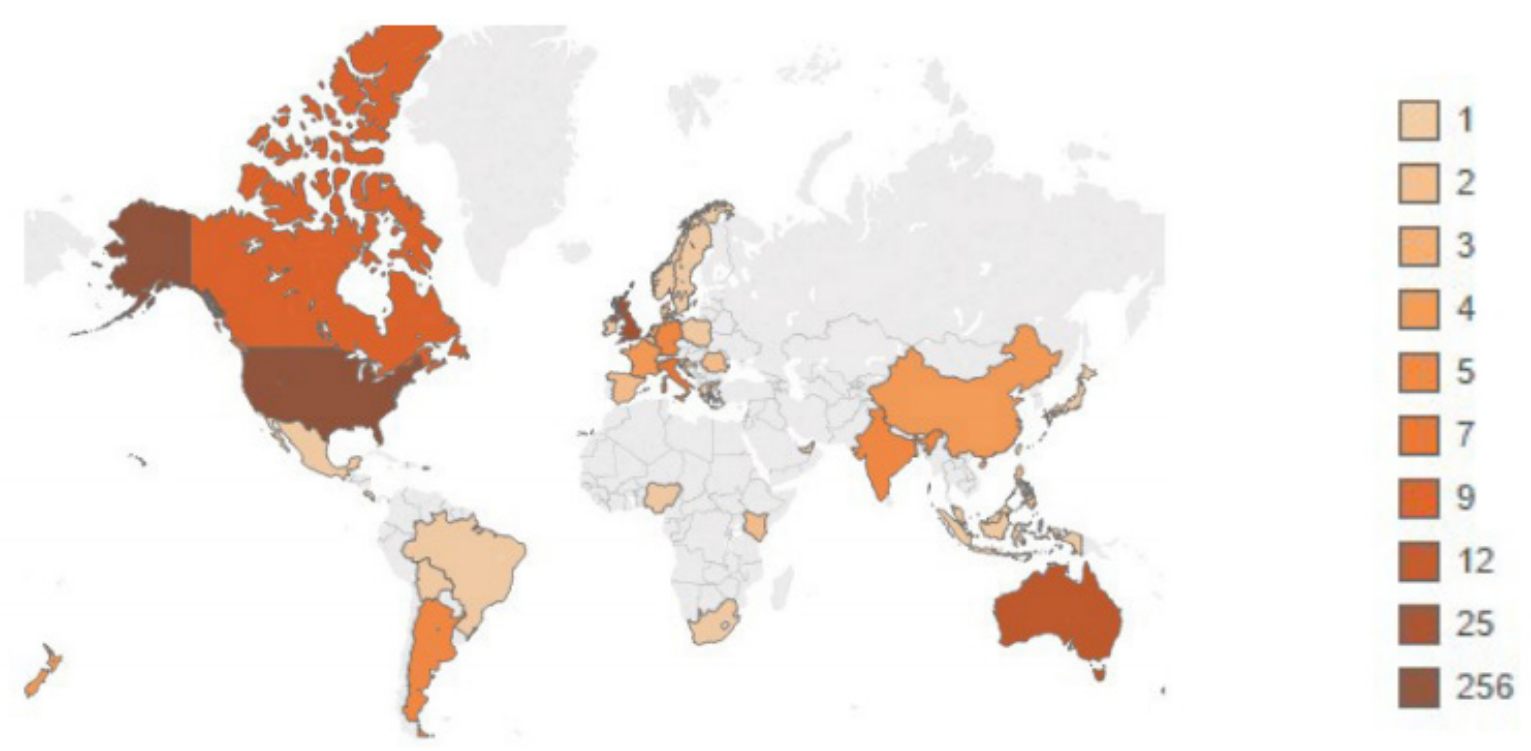

Figure 2. Worldwide distribution of the 372 patients with $\mathrm{CD}$ registered with the $\mathrm{CDCN}$

The second phase of the CDCN was focused on leveraging the community to prioritize research projects ${ }^{8}$. The CDCN reached out to their new collaborative community to gather information on what was currently known about the disease and what needed to be done in the future. The future projects were prioritized based on their feasibility and impact, with the idea that the entire global community agreed upon and would support this list. The third phase of the CDCN was to execute prioritized projects through proactive and reactive funding mechanisms ${ }^{8}$. Funding was sought from philanthropic and industry stakeholders and distributed to the top experts to carry out the studies that had been deemed high priority 8 Together, the CDCN has established a unified terminology system along with a new model of iMCD pathogenesis. Today, the CDCN includes a global network of over 300 physicians and researchers working together to advance the CDCN's community-prioritized international research agenda by sharing clinical data and tissue samples as well as conducting research. The CDCN has organized the four largest CD research meetings, hosted two international Castleman Disease Pathology workshops, and coordinated two summits that brought together $\mathrm{CD}$ patients and loved ones from around the world ${ }^{10}$.

The success of the CDCN has been a result of its focus on global collaboration. Herein, we report three mini-case examples of how global collaboration has helped to advance $\mathrm{CD}$ research, which we believe can serve as examples for other research networks and international researchers.

\section{Mini Case Report \#1: The Pathogen Discovery Study}

In iMCD, an inflammatory cascade occurs, but, as discussed above, the cause and drivers of the inflammatory cascade are unknown ${ }^{2}$. There are currently three hypotheses that may explain the pathologic hypercytokinemia that drives iMCD: autoimmune mechanisms, paraneoplastic secretion of proinflammatory cytokines by malignant cells, and infection with an unknown virus ${ }^{11}$. iMCD's overlapping clinical and laboratory features with HHV-8 MCD suggest that an unknown virus may be responsible for iMCD. Additionally, pathogens such as Hepatitis B and gamma-herpesvirus members Epstein-Barr Virus and Human Herpesvirus 6 have been reported in case reports associated with $\mathrm{iMCD}{ }^{11}$.

In order to investigate the hypothesis that a virus may be responsible for iMCD, the CDCN contracted with the Center for Infection and Immunity (CII) at Columbia University to perform a pathogen discovery study. Nucleic acids will be isolated from lymph nodes, the RNA pool will be reverse transcribed and PCR amplified to generate a sequencing library, and the sequencing libraries will then go through a pipeline to identify potential candidates ${ }^{11}$. 
Unfortunately, CII would need at least 25 lymph nodes samples from a variety of disease states to perform this study, and the lymph node samples must be flash-frozen since RNA is sensitive to degradation during fixation ${ }^{11}$. Lymph nodes are almost never frozen when removed clinically, and the $\mathrm{CDCN}$ was pessimistic about being able to identify enough samples for the study.

In response to this problem, the $\mathrm{CDCN}$ recognized the need for global collaboration in order to gather enough samples for the study. It turned to its international network of over 300 physicians and researchers and identified 34 viable samples from seven different institutions. Once the samples were located and the collaborators were on board with the study, the CDCN assisted with the logistics of Institutional Review Board (IRB) approvals, patient consent from the tissue donors, Material Transfer Agreements with both Columbia University and its collaborators, and coordination of shipping. Nearly all of the samples have arrived at Columbia University, and the CDCN expects to have data back on this key study by mid-2016. This potentially groundbreaking study could not have been performed by a single institution, as it required global collaboration.

\section{Mini Case Report \#2: The Global Patient Registry}

One of the greatest barriers to successfully treating iMCD has been the lack of a database to track treatments and patient care, leaving physicians to make care decisions with limited data. The CDCN aims to solve this problem by creating a global registry (ACCELERATE) that will collect real-world demographic, clinical, laboratory, patient-reported outcomes, and treatment data on patients with $\mathrm{CD}^{12}$. Such a registry could help to guide physicians in making more informed, evidence-based decisions when treating patients with $\mathrm{CD}$. The $\mathrm{CDCN}$ has engaged and partnered with a top pharmaceutical company to build this registry at the University of Pennsylvania ${ }^{12}$.

The registry will focus on enrolling patients from Europe and North America, but patients from anywhere around the world will be able to enroll as well. In Europe, patients will be enrolled by participating physicians from 10 study sites with medical record data entry by site staff and optional patient-reported data entry. In North America and other countries outside of Europe, patients will selfenroll, and the ACCELERATE Registry Team will perform medical record data entry.

The creation of this registry will benefit the international Castleman disease community in three important ways: it will track the natural history of the disease, track real-world treatment use and safety profiles, and build an infrastructure for future translational research by collecting clinical data and tissue samples ${ }^{13}$. The results of the registry will improve understanding of $\mathrm{CD}$ at the international level. A successful registry requires a community of physicians, researchers, and patients to work together to prioritize research questions, actively participate, and contribute data for the registry. The CDCN's international community, scientific advisory board (SAB) of experts, and strong patient community will enable the ACCELERATE team to recruit the number and type of patients needed for the registry ${ }^{8}$.

\section{Mini Case Report \#3: The Importance of Studying Regional Differences}

The pursuit of global collaboration is crucial in studying a rare disease, since similar pathology can present itself asynchronously in different parts of the world. Accordingly, Castleman disease clinical symptoms can vary across geographic regions. In a Japanese clinical trial, baseline measurements demonstrated that 17 of 26 had lymphocytic interstitial pneumonitis and 12 of 26 had violaceous, lymphocytic skin lesions ${ }^{1}$. These findings are notable, because patients with $\mathrm{MCD}$ in other regions almost never demonstrate these features. Recognizing these geographic differences is imperative in conducting sound scientific research.

Varied presentations across geographies are not unique to iMCD. Epstein-Barr Virus (EBV) is found widely across the world but presents itself in different ways in different regions of the world. The two types of EBV (1 and 2) differ in their geographic distribution ${ }^{6}$; Type 1 is most common as a whole across the world, but Type 2 is more prevalent in Africa, Papua New Guinea, and Alaska ${ }^{14}$. In Central Africa and Papua New Guinea, EBV-infected patients show the clinical condition of Burkitt's lymphoma, while their Asian counterparts show the clinical condition 
of nasopharyngeal carcinoma ${ }^{14}$. When approaching $\mathrm{CD}$, it is important to keep in mind that global collaboration is crucial in order to recognize potential etiological and pathological differences in CD across the world.

It is not known why East Asian patients (either born in East Asia or of East Asian ancestry born in other regions) express different clinical symptoms than other patient populations. One hypothesis accounting for the variation is genotype differences. An alternate hypothesis is the possibility of a different pathogen or subtype of a pathogen responsible for $\mathrm{CD}$ in different regions. Further research is needed to discover the cause for iMCD and address these clinical differences.

A collaborative global network is also important in order for physicians and researchers to quickly disseminate discoveries in other countries. In Japan, a subset of iMCD patients found to demonstrate similar symptoms was identified and described as "TAFRO" syndrome ${ }^{15}$. These patients demonstrated Thrombocytopenia, Anasarca, Fever, Reticulin fibrosis, and Organomegaly. Initially, researchers in Japan considered this subtype of $\mathrm{MMCD}$ to be unique to Japan. However, members of the CDCN posted on the group's online forum about cases in North America and throughout Europe with TAFRO syndrome, suggesting that these features can be seen outside of Japan. Compared to iMCD patients without TAFRO syndrome, iMCD patients with TAFRO syndrome have a more aggressive clinical course, and the CDCN is focused on studying this subtype in patients around the world.

\section{Challenges}

Global collaboration has been essential for improving the understanding and treatment of CD.

\section{REFERENCES}

1. Munshi N, Mehra M, van de Velde H, Desai A, Potluri R, Vermeulen J. Use of a claims database to characterize and estimate the incidence rate for Castleman disease. Leuk Lymphoma. 2015;56(5):1252-60. doi: $10.3109 / 10428194.2014 .953145$

2. Fajgenbaum DC, van Rhee F, Nabel CS. HHV-8negative, idiopathic multicentric Castleman disease: novel insights into biology, pathogenesis, and therapy. Blood. 2014;123(19):2924-33. doi: 10.1182/blood-2013-12-545087.

3. Nishimoto N, Kanakura Y, Aozasa K, Johkoh T, Nakamura M,
Nevertheless, there are several important challenges that come with global collaborations, such as language barriers and the use of different terminology systems to describe subtypes of the disease in different regions, which may cause misinterpretation. Furthermore, extended travel times for shipping samples between countries and inter-country shipping barriers can impede collaboration. Lastly, a global community is very difficult to connect for face-to-face meetings.

\section{CONCLUSION}

$\mathrm{CD}$ is a complicated and potentially fatal disease that requires global collaboration among physicians, researchers, and patients in order to improve the knowledge and treatment of the disease. Prior to the formation of the CDCN, there was little, if any, global collaboration in CD research, and the outlook for the disease was poor. The CDCN's emphasis on global collaboration has facilitated the advancement in understanding of CD in a variety of realms. A Pathogen Discovery Study was launched that could potentially explain the etiology of iMCD which could not have been possible at any one institution. The CDCN's global network provides the infrastructure needed to build a global registry and to enroll the requisite patients. Finally, the CDCN's global reach has helped to identify epidemiological and phenotypic differences and similarities in $\mathrm{CD}$ across regions that could never have been reviewed without the CDCN's network. Global collaboration has its challenges, but it also provides a great deal of hope for advancing understanding of $\mathrm{CD}$ and moving closer to a cure. We hope that the CDCN's model and these mini-case studies can be useful to other rare disease organizations to facilitate global collaboration.

Nakano S, Ikeda Y, Sasaki T, Nishioka K, Hara M, Taguchi H, Kimura Y, Kato Y, Asaoku H, Kumagai S, Kodama F, Nakahara H, Hagihara K, Yoshizaki K, Kishimoto T. Humanized anti-interleukin-6 receptor antibody treatment of multicentric Castleman disease. Blood. 2005;106(8):262732. doi: http://dx.doi.org/10.1182/blood-2004-12-4602.

4. Newman SK, Jayanthan RK, Mitchell GW, Carreras Tartak JA, Croglio MP, Suarez A, Liu AY, Razzo BM, Oyeniran E, Ruth JR, Fajgenbaum DC. Taking control of Castleman disease: Leveraging Precision Medicine Technologies to Accelerate Rare Disease Research. Yale J Biol Med. 
2015;88(4):383-8. Available from: http://www.ncbi.nlm.nih. gov/pmc/articles/PMC4654187/.

5. Gérard L, Bérezné A, Galicier L, et al. Prospective study of rituximab in chemotherapy-dependent human immunodeficiency virus-associated multicentric Castleman's disease: ANRS 117 CastlemaB Trial. J Clin Oncol. 2007;25(22):3350-6. doi: 10.1200/JCO.2007.10.6732.

6. Lilford RJ, Thornton JG, Braunholtz D. Clinical trials and rare diseases: a way out of a conundrum. BMJ. 1995;311(7020):1621-5. Available from: http://www.ncbi. nlm.nih.gov/pmc/articles/PMC2551510/pdf/bmj006230045.pdf.

7. van Rhee F, Wong RS, Munshi N, Rossi J-F, Ke X-Y, Fosså A, et al. Siltuximab for multicentric Castleman's disease: a randomised, double-blind, placebo-controlled trial. Lancet Oncol. 2014;15(9):966-74. doi: http://dx.doi.org/10.1016 ?S1470-2045(14)70319-5.

8. Fajgenbaum D, Ruth J, Kelleher D, Rubenstein A. the collaborative network approach: a new framework for accelerating Castleman disease and other rare disease research. Lancet Haematol. 2016;3(4):e150-2. doi: htp:// dx.doi.org/10.1016/S2352-3026(16)00007-7.

9. Tableau Public. 9.2.0. Seattle, Washington: Tableau Software; 2015.

10. Castleman Disease Collaborative Network (CDCN). CDCN hosts international CD pathology preceptorship with experts from Latin America. Paso Robles, CA; 2015 [cited 2015 Dec 28]. Available from: http://www.cden.org/news-events/ cden-hosts-international-cd-pathology-preceptorship-withexperts-from-latin-america.

11. Nabel CS. Castleman Disease Collaborative Network seed grant RFP. A Pilot Study in Pathogen Discovery in idiopathic Multicentric Castleman Disease; Perelman School of Medicine. Philadelphia, PA; 2015.

12. Fajgenbaum DC. ACCELERATE: an international registry for patients with Castleman disease. Philadelphia, PA: University of Pennsylvania; 2015. p.1-42.

13. Three Global Leaders Join Together to ACCELERATE Care for a Rare and Deadly Disease [internal report]. Philadelphia, PA: Castleman Disease Collaborative Network; 2016.

14. Arvin A, Campadelli-Fiume G, Mocarski E, Moore PS, Roizman B, Whitley R, et al. The epidemiology of EBV and its association with malignant disease. In: Arvin A, CampadelliFiume G, Mocarski E, et al., editors. Human herpesviruses: biology, therapy, and immunoprophylaxis. Cambridge, MA: Cambridge University Press; 2007. p.929-59.

15. Iwaki N, Fajgenbaum DC, Nabel CS, Gion Y, Kondo E, Kawano M, et al. Clinicopathologic analysis of TAFRO syndrome demonstrates a distinct subtype of HHV-8negative multicentric Castleman disease. Am J Hematol. 2016;91(2):220-6. doi: 10.1002/ajh.24242. 